\title{
Lifetime alcohol abstainers and moderate drinkers have a lower lifetime prevalence of mood and anxiety disorders than problem drinkers
}

Sareen J, McWilliams L, Cox B, et al. Does a U-shaped relationship exist between alcohol use and DSM-III-R mood and anxiety disorders? J Affect Disord 2004;82:113-18.

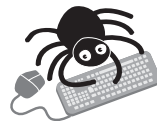

This article contains extra text on the EBMH website

Is the lifetime prevalence of mood and anxiety disorders similarly high in people with high lifetime levels of alcohol consumption and in lifetime alcohol abstainers?

\section{METHODS}

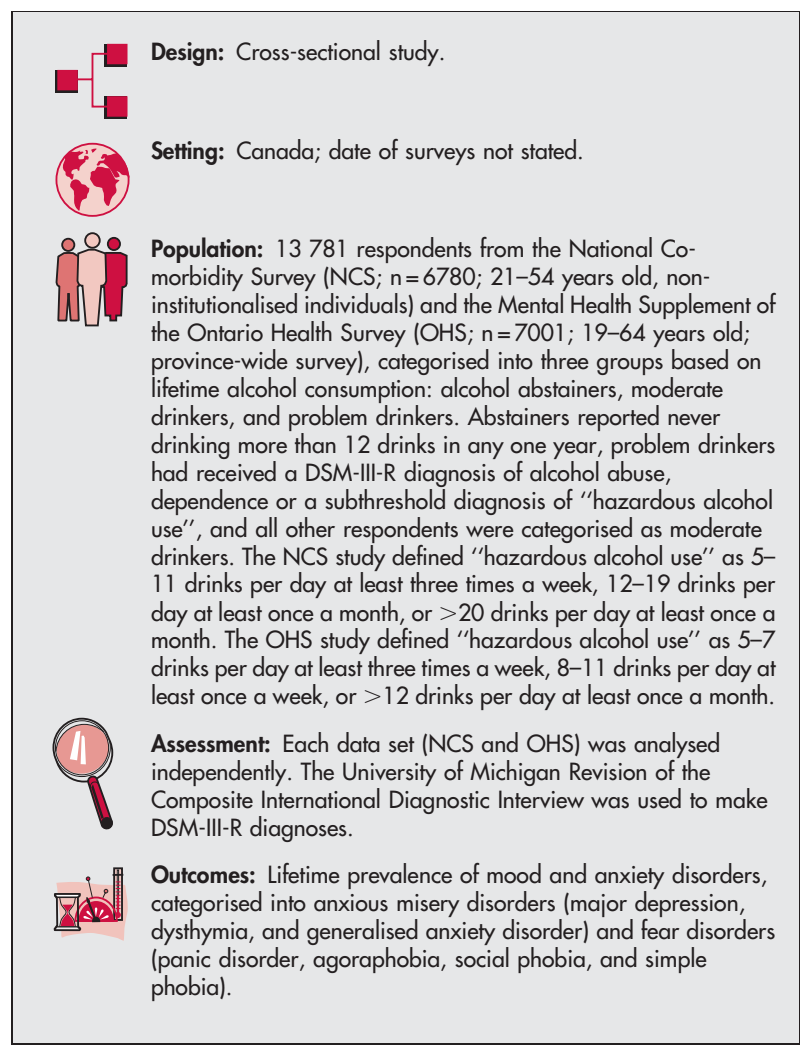

\section{MAIN RESULTS}

Problem drinkers had the highest lifetime prevalence of both anxious misery disorders (28.4\% NCS, $17.4 \%$ OHS) and fear disorders $(30.0 \%$ NCS, $26.3 \%$ OHS). Moderate drinkers and abstainers had a similar lifetime prevalence of anxious misery disorders and fear disorders (see http://www.ebmentalhealth.com/supplemental for table).

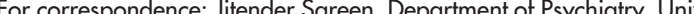
Manitoba, PZ203-771 Bannatyne Avenue, Winnipeg, Manitoba, Canada R3E 3N4; sareen@cc.umanitoba.ca

Sources of funding: Ontario Government (Ontario Health Survey Mental Health Supplement), National Institute of Mental Health, National Institute of Drug and Alcohol Abuse, and the WT Grant Foundation (National Comorbidity Survey).

\section{CONCLUSIONS}

There is no evidence that the lifetime prevalence of mood and anxiety disorders in alcohol abstainers is as high as in problem drinkers.

Commentary

wo well known previous epidemiologic surveys ${ }^{12}$ have reported that, compared with moderate drinkers, people with excessive alcohol consumption and people who abstain from alcohol have an increased prevalence of depressive and anxiety symptoms-a so called "U shaped" relationship. In their very interesting, brief report Sareen et al examined whether a $U$ shaped relationship exists between the lifetime pattern of alcohol use and lifetime prevalence of mood and anxiety disorders. Somewhat discordantly, Sareen et al find that abstainers have no relative excess in diagnosed mood and anxiety disorders compared with moderate drinkers, but almost the contrary.

Why do the findings of these studies apparently differ? The results most likely reflect important conceptual and methodological differences between the studies. Sareen et al used a validated diagnostic measure (DSM-III-R) to identify people with mood and anxiety disorders, while the two previous studies simply analysed the presence of self-reported symptoms of depression and anxiety. In addition, the studies differ in their definition of the abstinent population. Sareen et al classified people according to their lifetime alcohol consumption while the previous two studies used current alcohol consumption. Therefore, a person who had been alcohol dependent in the past but was currently abstinent would be categorised as a "problem drinker" in the Sareen study but as abstinent in the previous two studies. It seems likely that people in remission from alcohol dependence would be different to a lifetime abstinent population with regard to psychiatric symptoms and disorders, and therefore the approach used by Sareen et al may be more appropriate.

The findings of Sareen et al are interesting, and add to our knowledge of an area where there is little research. However, the study does have important limitations. The accuracy of the findings are likely to have been adversely affected by retrospective data collection, with recall bias and perceived stigma leading to underreporting of both drinking and psychiatric diagnoses. In addition, the high rate of abstinence among the general population in North America may limit the generalisability of these findings to countries with less abstinent populations. Finally, the cross-sectional design of the study means that causal links cannot be identified between alcohol intake and psychiatric diagnoses. Prospective cohort studies are needed to further examine this relationship.

Sami Pirkola, MD, PhD

Project Manager, Mental Health Group of National Research and Development Centre for Welfare and Health, and Senior Researcher, the Department of Mental Health and Alcohol Research of the National Public Health Institute, Finland

1 Power C, Rodgers B, Hope S. U-shaped relation for alcohol consumption and health in early adulthood and implications for mortality. Lancet 1998:352:877.

2 Rodgers B, Korten AE, Jorm AF, et al. Non-linear relationships in associations of depression and anxiety with alcohol use. Psychol Med 2000;30:421-32. 